International Journal of Pure and Applied Mathematics

Volume 115 No. $4 \quad 2017,859-865$

ISSN: 1311-8080 (printed version); ISSN: 1314-3395 (on-line version)

url: http://www.ijpam.eu

doi: 10.12732/ijpam.v115i4.19

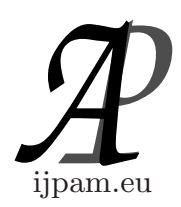

\title{
COMPUTING SANSKRUTI INDEX OF V-PHENYLENIC NANOTUBES AND NANOTORI
}

\author{
H. Jiang ${ }^{1}$, M.S. Sardar ${ }^{2}$, M.R. Farahani ${ }^{3} \S$, \\ M. Rezaei ${ }^{4}$, M.K. Siddiqui ${ }^{5}$ \\ ${ }^{1}$ School of Information Science and Engineering \\ Chengdu University \\ Chengdu, 610106, P.R. CHINA \\ ${ }^{2}$ University of Management and Technology (UMT) \\ Lahore, PAKISTAN \\ ${ }^{3}$ Department of Applied Mathematics \\ Iran University of Science and Technology (IUST) \\ Narmak, Tehran, 16844, IRAN \\ ${ }^{4}$ Department of Mathematics \\ Buein Zahra Technical University \\ Buein Zahra, Qazvin, IRAN \\ Department of Mathematics \\ ${ }^{5}$ Comsats Institute of Information Technology \\ Sahiwal, PAKISTAN
}

\begin{abstract}
Among topological descriptors connectivity topological indices are very important and they have a prominent role in chemistry. One of them is Sanskruti index defined as $S(G)=\sum_{u v \in E(G)}\left(\frac{S_{u} S_{v}}{S_{u}+S_{v}-2}\right)^{3}$ where $S_{u}$ is the summation of degrees of all neighbors of vertex $u$ in $G$. In this paper we compute this topological index for V-phenylenic nanotube and nanotori.
\end{abstract}

AMS Subject Classification: 05C90, 05C35, 05C12

Key Words: molecular graph, benzenoid, Capra operation, topological index, Sanskruti index

Received: $\quad$ May 4, 2017

Revised: July 10, 2017

Published: August 9, 2017

$\S_{\text {Correspondence author }}$ (c) 2017 Academic Publications, Ltd. url: www.acadpubl.eu 


\section{Introduction and Preliminaries}

Let $G=(V ; E)$ be a simple molecular graph without directed and multiple edges and without loops, the vertex and edge sets of it are represented by $V=V(G)$ and $E=E(G)$, respectively. In chemical graphs, the vertices correspond to the atoms of the molecule, and the edges represent to the chemical bonds. Also, if $e$ is an edge of $G$, connecting the vertices $u$ and $v$, then we write $e=u v$ and say $u$ and $v$ are adjacent.

Mathematical chemistry is a branch of theoretical chemistry for discussion and prediction of the molecular structure using mathematical methods without necessarily referring to quantum mechanics. Chemical graph theory is a branch of mathematical chemistry which applies graph theory to mathematical modeling of chemical phenomena ([1]-[5]). This theory had an important effect on the development of the chemical sciences.

Among topological descriptors, connectivity indices are very important and they have a prominent role in chemistry. In other words, if $G$ be the connected graph, then we can introduce many connectivity topological indices for it, by distinct and different definition. A connected graph is a graph such that there is a path between all pairs of vertices. One of the best known and widely used is the connectivity index, introduced in 1975 by Milan Randic [6], who has shown this index to reflect molecular branching and defined as follows:

$$
R(G)=\sum_{u v \in E(G)} \frac{1}{\sqrt{d_{u} d_{v}}}
$$

The Sanskruti index $S(G)$ of a graph $G$ is defined as follows (see [7]-[11]):

$$
S(G)=\sum_{u v \in E(G)}\left(\frac{S_{u} S_{v}}{S_{u}+S_{v}-2}\right)^{3} .
$$

where $S_{u}$ is the summation of degrees of all neighbors of vertex $u$ in $G$. In Refs [12]-[27] some topological indices of $V$-phenylenic nanotube and $V$-phenylenic nanotori are computed. In this paper, we continue this work to compute the Sanskruti index of molecular graphs related to $V$-phenylenic nanotube and nanotori. Our notation is standard and mainly taken from Refs. [1]-[5].

\section{Main Results and Discussion}

The goal of this section is to computing the Sanskruti index of $V$ - phenylenic nanotube and nanotori. The novel phenylenic and naphthylenic lattices pro- 
posed can be constructed from a square net embedded on the toroidal surface. Phenylenes are polycyclic conjugated molecules, composed of four membered ring (=square) and six-membered rings (=hexagons) such that every four membered ring (4-membered cycle) is adjacent to two 6-membered cycles, and no two six-membered rings are mutually adjacent. Each four-membered ring lies between two six-membered rings, and each hexagon is adjacent only two fourmembered rings. Because of such structural features phenylenes are very interesting conjugated species [28]-[33]. The rapid development of the experimental study of phenylenes motivated a number of recent theoretical studies of thee conjugated $\pi$-electron systems [33].

Following M. V. Diudea [5] we denote a $V$-Phenylenic nanotube and $V$ Phenylenic nanotorus by $G=V P H X[m, n]$ and $H=V P H Y[m, n]$, respectively. The general representation of these nano structures are shown in Figure 1 and Figure 2. For more information and background materials, refer to paper series [12]-[33] again. Now we have following theorems, immediately.

Theorem 2.1. $\forall m, n \in N$, the Sanskruti index $S(G)$ of $V-$ Phenylenic Nanotube $\operatorname{VPHX}[m, n]$ is equal to

$$
S(V P H X[m, n])=-\frac{87714218531}{175616000} m+\frac{4782969}{4096} m n .
$$

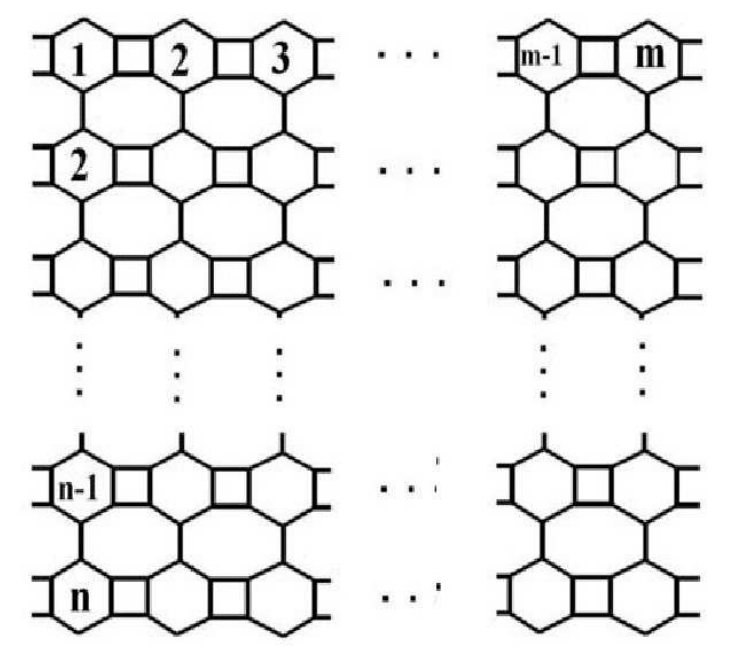

Figure 1: The Molecular Graph of $V$-Phenylenic Nanotube $V P H X[m, n]$. 
Proof. Consider the $V$-phenylenic nanotube $G=V P H X[m, n]$ with $6 m n$ vertices and $9 m n-m$ edges (Figure 1 ). In $V$ - phenylenic molecule, there are two partitions $V_{2}=v \in V(G) \mid d_{v}=2$ and $V_{3}=v \in V(G) \mid d_{v}=3$ of $V(V P H X[m, n])$, since the degree of an arbitrary vertex/ atom of a molecular graph is equal to 2 or 3 . Next, the two partitions of $E(G)$ are $E_{5}=\{u, v \in$ $\left.V(G) \mid d_{u}=3 \& d_{v}=2\right\}$ and $E_{6}=\left\{u, v \in V(G) \mid d_{u}=d_{v}=3\right\}$.

Also, two adjacent vertices $v_{1}, v_{2}$ of a vertex $v \in V 2$ have degree three, then $S_{v}=2 \times 3=6$ and two edges $v v_{1}$ and $v v_{2}$ belong to $E_{5}\left(\right.$ and $\left|E_{5}\right|=2\left|V_{2}\right|=4 m$ ). Also, for all vertices $u$ in first and end row of $V$-phenylenic nanotube with degree three, $N(u)=v_{1}, v_{2}, v_{3}$ such that $v_{1} \in V_{2}$ and $v_{2}, v_{3} \in V_{3}\left(u v_{1} \in E_{5}\right.$ and $\left.u v_{3}, u v_{2} \in E 6\right)$, thus $S_{u}=2 \times 3+2=8$. Finally, for other vertices $S_{w}=9$, because all other vertices and their edges belong to $V_{3}$ and $E_{6}$, respectively. So, the Sanskruti index $S(G)$ of $V P H X[m, n](m, n \geq 1)$ will be

$$
\begin{aligned}
S(V P H X[m, n]= & \sum_{u v \in E(G)}\left(\frac{S_{u} S_{v}}{S_{u}+S_{v}-2}\right)^{3} \\
= & 4 m \cdot\left(\frac{6.8}{6+8-2}\right)^{3}+2 m \cdot\left(\frac{8.8}{8+8-2}\right)^{3}+2 m \cdot\left(\frac{8.9}{8+9-2}\right)^{3} \\
& +(9 m n-9 m) \cdot\left(\frac{9.9}{9+9-2}\right)^{3} \\
= & -\frac{87714218531}{175616000} m+\frac{4782969}{4096} m n .
\end{aligned}
$$

Theorem 2.2. $\forall m, n \in N$, the Sanskruti index $S(G)$ of $V$ - Phenylenic Nanotori $H=V P H Y[m, n]$ is equal to

$$
S(V P H Y[m, n])=\frac{4782969}{4096} m n .
$$

Proof. The proof is easily, since by considering the $V$-phenylenic nanotori $H=V P H Y[m, n]$ with $6 m n$ vertices and $9 m n$ edges (Figure 2). We see that this nanotori is a Cubic graph and all vertices belong to $V_{3}$ and $\forall v \in V(V P H Y[m, n]) S_{v}=9$. This implies that all edges belong

$$
\begin{aligned}
S(V P H X[m, n] & =\sum_{u v \in E(G)}\left(\frac{S_{u} S_{v}}{S_{u}+S_{v}-2}\right)^{3} \\
& =(9 m n) \cdot\left(\frac{9.9}{9+9-2}\right)^{3}=\frac{4782969}{4096} m n .
\end{aligned}
$$




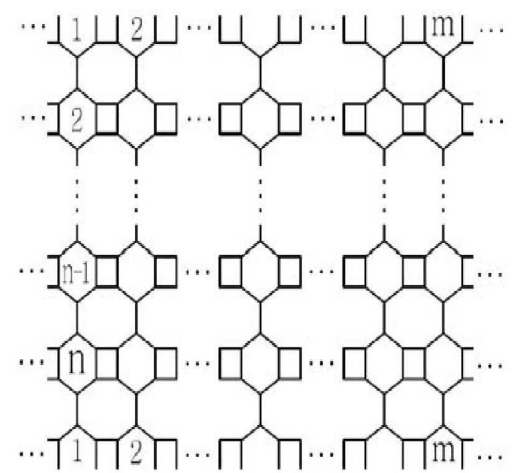

Figure 2: The Molecular Graph of V-Phenylenic Nanotorus $V P H Y[m, n]$.

\section{Conclusions}

In this report, we study some properties of a new connectivity index of (molecular) graphs that called Sanskruti index. This connectivity index was defined as follows:

$$
S(G)=\sum_{u v \in E(G)}\left(\frac{S_{u} S_{v}}{S_{u}+S_{v}-2}\right)^{3} .
$$

where $S_{u}$ is the summation of degrees of all neighbors of vertex $u$ in $G$. In continue, closed analytical formulas for $S(G)$ of a physico chemical structure of phenylenic nanotubes and nanotorus are given. These nano structures are $V$-Phenylenic Nanotube $V P H X[m, n]$ and $V$-phenylenic nanotorus $V P H Y[m$, $n]$.

\section{References}

[1] R. Todeschini and V. Consonni: Handbook of Molecular Descriptors, Wiley-TUACH, Weinheim (2000).

[2] N. Trinajstic: Chemical Graph Theory, 2nd ed. CRC Press; Boca Raton, FL: (1992); 20-75.

[3] I. Gutman, N. Trinajstic: Graph theory and molecular orbitals. Total felectron energy of alternant hydrocarbons. Chem. Phys. Lett. 17(4), (1972), 535-538. https://doi.org/10.1016/0009-2614(72)85099-1

[4] P. J. Cameron: Cambridge University Press, Cambridge, (1994).

[5] M. V. Diudea: Fuller. Nanotub. Carbon Nanostruct, (2002), 10, 273-292. 
[6] M. Randic: Characterization of molecular branching. J. Amer. Chem. Soc. 97, (1975) 6609-6615. DOI: 10.1021/ja00856a001

[7] S. M. Hosamani: S. M. Hosamani: Computing Sanskruti index of certain nanostructures. Journal of Applied Mathematics and Computing. In press, 2017. DOI 10.1007/s12190-016-1016-9.

[8] M.S. Sardar, S. Zafar, M.R. Farahani. Computing Sanskruti index of the Polycyclic Aromatic Hydrocarbons. Geology, Ecology, and Landscapes. In press, (2017), http : //dx.doi.org/10.1080/24749508.2017.1301056

[9] Y.Y. Gao, M.R. Farahani, M.S. Sardar, S. Zafar. On the Sanskruti Index of Circumcoronene Series of Benzenoid. Applied Mathematics. 8 (4), (2017), 520-524. doi:10.4236/am.2017.84041

[10] Y.Y. Gao, M.S. Sardar, S.M. Hosamani, M.R. Farahani. Computing sanskruti index of $T U R C_{4} C_{8}(s)$ nanotube. International Journal of Pharmaceutical Sciences and Research. 8 (10), (2017), 1000-03. doi: 10.13040/IJPSR.0975-8232.8(10).1000-03.

[11] Y.Y. GaO, M.S. Sardar, S. Zafar, M.R. Farahani. Computing Sanskruti index of dendrimer nanostars. International Journal of Pure and Applied Mathematics. 115(2), In press, (2017), http://ijpam.eu/contents/index.php

[12] V. Alamian, A. Bahrami and B. Edalatzadeh: PI Polynomial of V-Phenylenic Nanotubes and Nanotori. Int. J. Mol. Sci., 9(3), (2008), 229-234; doi:10.3390/ijms9030229

[13] M. Alaeiyan, A. Bahrami and M. R. Farahani: Cyclically Domination Polynomial of Molecular Graph of Some Nanotubes. Digest. J. Nanomater. Bios. 6(1), (2011), 143-147.

[14] J. Asadpour: Some topological polynomial indices of nanostructures, Optoelectron. Adv. Mater.-Rapid Commun. 5(7), (2011), 769-772.

[15] A. Bahrami and J. Yazdani: Vertex PI Index of V-Phenylenic Nanotubes and Nanotori. Digest Journal of Nanomaterials and Biostructures, 4(1), (2009), 141-144.

[16] M. Davoudi Mohfared, A. Bahrami and J. Yazdani: PI Polynomial of V-Phenylenic Nanotubes. Digest Journal of Nanomaterials and Biostructures, 5(2), (2010), 441-445.

[17] M. R. Farahani: Computing $G A_{5}$ index of V-Phenylenic Nanotubes and Nanotori. Int. J. Chem Model. 5(4), (2013), 479-484.

[18] M. R. Farahani: Computing fourth atom-bond connectivity index of V-Phenylenic Nanotubes and Nanotori. Acta Chimica Slovenica. 60(2), (2013), 429-432.

[19] M. R. Farahani: omputing Theta Polynomial and Theta Index of V-phenylenic Planar, Nanotubes and Nanotoris. Int. J. Theoretical Chemistry. 1(1), (2013), 01-09.

[20] M.R. Farahani, M.R. Rajesh Kanna: The Generalized Zagreb Index of V-Phenylenic Nanotubes and Nanotorus. Journal of Chemical and Pharmaceutical Research. 7(11), (2015), 241-245.

[21] M. Ghorbani, H. Mesgarani, S. Shakeraneh: Computing GA index and ABC index of V-phenylenic nanotube. Optoelectron. Adv. Mater.-Rapid Commun. 5(3), (2011), 324326.

[22] A. D. Manikpuri, S. Aziz, E. John, P.V. Khadikar: Computation of the Sadhana (Sd) Index of Linear Phenylenes and Corresponding Hexagonal Sequences. Iranian J. Math. Chem, 1(1), (2010), 79-90. 
[23] P.E. John, S. Aziz, P.V. Khadikar: Use of Structure Codes (Counts) for Computing Topological Indices of Carbon Nanotubes: Sadhana (Sd) Index of Phenylenes and its Hexagonal Squeezes. Iranian J. Math. Chem, 1(1), (2010), 91-94.

[24] Z. Yarahmadi: A Note on the First Geometric-Arithmetic Index of Hexagonal Systems and Phenylenes. Iranian J. Math. Chem, 2(2), (2011), 101-108.

[25] S. Moradi, S. Babarahimi and M. Ghorbani: Two Types of Geometric-Arithmetic index of V-phenylenic Nanotube. Iranian J. Math. Chem, 2(2), (2011), 109-117.

[26] M. Ghorbani, H. Mesgarani, S. Shakeraneh: Optoelectron. Adv. Mater. Rapid Commun. 2011, 5(3), 324-326.

[27] N. Prabhakara Rao and K. L. Lakshmi: Eccentricity Connectivity Index of VPhenylenic Nanotubes. Digest Journal of Nanomaterials and Biostructures, 6(1), (2010), 81-87.

[28] S.C. Basak, Q. Zhu AND D. Mills: Prediction of Anticancer Activity of 2phenylindoles: Comparative Molecular Field Analysis Versus Ridge Regression using Mathematical Molecular Descriptors. Acta Chim. Slov. 57, (2010), 541-550.

[29] S. J. Cyvin and I. Gutman: Lecture Note in Chemistry, Springer-Verlag, Berlin, (1988), 46.

[30] I. Gutman and S. J. Cyvin: The number of Kekul, structures in long benzenoid chains. J. Chem. Phys. 1988, 147(1), 121-125. https://doi.org/10.1016/0009-2614(88)80235-5

[31] I. Gutman And S. J. Cyvin: Counting Kekule structures in some conjugated systems with repeating units. J. Serb. Chem. Soc. 1990, 55, 555-561.

[32] I. Gutman, S. Markovic, V. Lukovic, V. Radivojevic and S. Randić Coll. Sci. Papers Fac. Sci. Kragujevac, (1987), 8, 15-34.

[33] I. Gutman, P. Petkovic and P. V. Khadikar: Bounds for the total $\pi$-electron energy of phenylenes. Revue Roumaine de Chimie. 41, (1996), 637-643. 
